\title{
Blood Oxygenation Level-Dependent Visualization of Synaptic Relay Stations of Sensory Pathways along the Neuroaxis in Response to Graded Sensory Stimulation of a Limb
}

\author{
Johan Lilja, ${ }^{1}$ Toshiki Endo, ${ }^{1}$ Christoph Hofstetter, ${ }^{1}$ Eric Westman, ${ }^{2}$ Jeremy Young, ${ }^{4}$ Lars Olson, ${ }^{1}$ and Christian Spenger ${ }^{3}$ \\ ${ }^{1}$ Department of Neuroscience, ${ }^{2}$ Neurotec, and ${ }^{3}$ Clintec, Karolinska Institutet, S-171 76 Stockholm, Sweden, and ${ }^{4}$ AstraZeneca R\&D, 15185 Södertälje, \\ Sweden
}

Blood oxygenation level-dependent (BOLD) functional magnetic resonance imaging (fMRI) was used to test at which levels of the neuroaxis signals are elicited when different modalities of sensory information from the limbs ascend to cortex cerebri. We applied graded electric stimuli to the rat hindlimbs and used echo-planar imaging to monitor activity changes in the lumbar spinal cord and medulla oblongata, where primary afferents of painful and nonpainful sensation synapse, respectively. BOLD signals were detected in ipsilateral lumbar spinal cord gray matter using sufficiently strong stimuli. Using stimuli well below the threshold needed for signals to be elicited in the spinal cord, we found BOLD responses in dorsal medulla oblongata. The distribution of these signals is compatible with the neuroanatomy of the respective synaptic relay stations of the corresponding sensory pathways. Hence, the sensory pathways conducting painful and nonpainful information were successfully distinguished. The fMRI signals in the spinal cord were markedly decreased by morphine, and these effects were counteracted by naloxone. We conclude that fMRI can be used as a reliable and valid method to monitor neuronal activity in the rat spinal cord and medulla oblongata in response to sensory stimuli. Previously, we also documented BOLD signals from thalamus and cortex. Thus, BOLD responses can be elicited at all principal synaptic relay stations along the neuroaxis from lumbar spinal cord to sensory cortex. Rat spinal cord fMRI should become a useful tool in experimental spinal cord injury and pain research.

Key words: fMRI; rat; spinal cord; somatosensory; dorsal horn; medulla; pain; morphine; BOLD; EPI

\section{Introduction}

Functional magnetic resonance imaging (fMRI) is widely used to investigate brain function during different motor tasks and various sensory stimulations in humans (Fransson et al., 1998, 1999; Hofbauer et al., 2004, 2006; Hwang et al., 2005; Olausson et al., 2005; Seung et al., 2005; Elfgren et al., 2006; Pastor et al., 2006). The most commonly used technique relies on blood oxygenation level-dependent (BOLD) contrast, which is indirectly related to neuronal activity (Ogawa et al., 1993; Heeger and Ress, 2002; Thompson et al., 2003; Mukamel et al., 2005; Niessing et al., 2005).

Studies of rodents have been instrumental in the development of fMRI (Silva et al., 1999). Although the small size of the rodent

\footnotetext{
Received Feb. 13, 2006; revised May 2, 2006; accepted May 2, 2006.

This work was supported by AFA Försäkring, the Swedish Research Council, Swedish Brain Power, "Karolinska Institutets Fonder," The National Institute on Drug Abuse, The Scandinavia-Japan Sasakawa Foundation, Stiftelse för Gamla Tjänarinnor, and Gun och Bertil Stohnes Stiftelse. The MRI and fMRI studies were performed at the Karolinska Experimental MRI unit, which is a core facility supported by the Karolinska University Hospital and Karolinska Institutet.

Correspondence should be addressed to Christian Spenger, Clintec, Experimental MR Research Center, Karolinska University Hospital, S-171 76 Stockholm, Sweden. E-mail: Christian.spenger@ki.se.

D01:10.1523/JNEUROSCI.0626-06.2006

Copyright $\odot 2006$ Society for Neuroscience $\quad$ 0270-6474/06/266330-07\$15.00/0
}

brain initially made fMRI relatively challenging, recent progress in MR hardware and scanning sequences has overcome these difficulties to allow functional mapping of the brain in response to peripheral sensory stimulation (Spenger et al., 2000; Keilholz et al., 2004). Introduction of $\alpha$-chloralose to animal anesthesia has helped to standardize the technique (Bock et al., 1998; Austin et al., 2005). In parallel, fMRI has been applied to animal models of spinal cord injury and stroke (Dijkhuizen et al., 2003; Hofstetter et al., 2003, 2005) and used to monitor pain perception (Tuor et al., 2000; Chang and Shyu, 2001).

Recently, fMRI of the spinal cord has been shown technically feasible in both humans and rats (Malisza et al., 2003; Stroman et al., 2003b; Majcher et al., 2006). In humans, the establishment of this technique should allow noninvasive assessment of spinal cord activity. It should also become valuable in spinal cord injury as a diagnostic tool and to monitor effectiveness of treatment and rehabilitation (Stroman et al., 2004). In rodents, monitoring distinct sensory systems using spinal cord fMRI could be valuable in spinal cord injury research. Moreover, fMRI should become useful in pain research and the understanding of mechanisms of central pain and the development of drugs to alleviate it.

Electrical stimulation has been widely used for fMRI studies in rodents (Spenger et al., 2000; Keilholz et al., 2004). Cutaneous 
Table 1. BOLD signals in the lumbar spinal cord in response to graded electrical stimulation of the hindlimb

\begin{tabular}{|c|c|c|c|c|c|c|c|c|c|c|c|c|}
\hline \multirow[b]{2}{*}{ Animal } & \multicolumn{2}{|c|}{$0.5 \mathrm{~mA} \alpha$-chloralose } & \multicolumn{2}{|c|}{$1.0 \mathrm{~mA} \alpha$-chloralose } & \multicolumn{2}{|c|}{$1.5 \mathrm{~mA} \alpha$-chloralose } & \multicolumn{2}{|c|}{$2.0 \mathrm{~mA} \alpha$-chloralose } & \multicolumn{2}{|c|}{$\begin{array}{l}2.0 \mathrm{~mA} \alpha \text {-chloralose } \\
\text { plus morphine }\end{array}$} & \multicolumn{2}{|c|}{$\begin{array}{l}2.0 \mathrm{~mA} \alpha \text {-chloralose } \\
\text { plus morphine plus } \\
\text { naloxone }\end{array}$} \\
\hline & $\beta$ estimate & $\begin{array}{l}\text { Cluster } \\
\text { volume }\end{array}$ & $\beta$ estimate & $\begin{array}{l}\text { Cluster } \\
\text { volume }\end{array}$ & $\beta$ estimate & $\begin{array}{l}\text { Cluster } \\
\text { volume }\end{array}$ & $\beta$ estimate & $\begin{array}{l}\text { Cluster } \\
\text { volume }\end{array}$ & $\beta$ estimate & $\begin{array}{l}\text { Cluster } \\
\text { volume }\end{array}$ & $\beta$ estimate & $\begin{array}{l}\text { Cluster } \\
\text { volume }\end{array}$ \\
\hline 1 & 0.22 & 0 & & & & & 1.95 & 2.46 & 1.74 & 2.05 & 1.80 & 2.26 \\
\hline 2 & 0.07 & 0 & & & & & 3.17 & 8.00 & 2.34 & 5.33 & 3.67 & 6.15 \\
\hline 3 & & & & & 2.63 & 0.62 & 2.64 & 1.64 & 0.51 & 0 & & \\
\hline 4 & & & & & 2.09 & 1.43 & 2.45 & 0.62 & 1.37 & 0.82 & & \\
\hline 5 & & & 0.82 & 0 & & & 3.04 & 0.62 & 2.46 & 0 & & \\
\hline 6 & & & 0.61 & 0 & & & 5.12 & 5.33 & 3.97 & 3.69 & 5.26 & 3.49 \\
\hline 7 & & & 1.01 & 0 & & & 3.74 & 4.31 & 1.65 & 0.82 & 1.65 & 1.44 \\
\hline 8 & & & 1.24 & 0 & & & 4.02 & 5.13 & 2.47 & 1.64 & 2.02 & 0.82 \\
\hline 9 & & & 1.29 & 0 & & & 5.35 & 2.46 & 4.08 & 1.44 & & \\
\hline 10 & & & 0.62 & 0 & & & 5.30 & 0.21 & 4.13 & 0 & & \\
\hline 11 & & & & & & & 7.40 & 15.6 & 5.41 & 10.1 & 6.66 & 16.2 \\
\hline 12 & & & & & & & 2.64 & 1.23 & 0.73 & 0.41 & 1.40 & 1.23 \\
\hline 13 & & & & & & & 3.93 & 7.79 & 0.80 & 5.54 & 2.53 & 8.61 \\
\hline 14 & 0.44 & 0 & & & 1.81 & 2.87 & 3.93 & 3.69 & 3.48 & 2.46 & 4.82 & 3.90 \\
\hline Mean & 0.24 & 0 & 0.93 & 0 & 2.18 & 1.64 & 3.91 & 4.22 & 2.51 & 2.45 & 3.31 & 4.90 \\
\hline SEM & \pm 0.11 & \pm 0 & \pm 0.11 & \pm 0 & \pm 0.24 & \pm 0.66 & \pm 0.39 & \pm 1.11 & \pm 0.40 & \pm 0.76 & \pm 0.63 & \pm 1.65 \\
\hline
\end{tabular}

$\beta$ estimate and cluster volume of the fMRI signal measured in the lumbar spinal cord in the slice with maximal intensity for animals $1-14$. Electric stimulation of the hindlimb was performed using $0.5,1.0,1.5$, or $2.0 \mathrm{~mA}$ current pulses. A block design with three conditions (alternating 2 different current levels and rest periods) was used in rats 1-10. A block design with two conditions (alternating a single current level with rest periods) was used for rats $11-14$. Rat 14 underwent $0.5,1.5$, and $2.0 \mathrm{~mA}$ stimulation with a block design with two conditions to generate Figure 2 . All animals were investigated under $\alpha$-chloralose anesthesia. Effects of morphine were assessed beginning 10 minutes after intravenous injection of morphine in a dose of $10 \mathrm{mg} / \mathrm{kg}$. Naloxone effects were assessed in the consecutive session and 5 minutes after the injection of naloxone in a dose of $4 \mathrm{mg} / \mathrm{kg}$.

sensory fibers mediating touch have low thresholds, whereas nociceptive afferent fibers have higher thresholds (Chang and Shyu, 2001). We hypothesized that weak stimuli, causing nonpainful sensations, would activate fibers mediating touch and therefore lead to BOLD signals in medulla oblongata, the first synaptic relay stations of the dorsal column pathway (Williams and Warwick, 1980), but not in the spinal cord. In contrast, stronger stimuli should lead to BOLD signals in the spinal cord where the nociceptive fibers synapse on the projection neurons of the spinothalamic tract.

The purpose of the current paper was (1) to establish BOLD fMRI of the rat lumbar spinal cord using echo-planar imaging (EPI) sequences to detect neuronal activity in response to peripheral electrical stimulation, (2) to test whether the method could differentiate between the dorsal column somatosensory pathway and the spinothalamic pathway, and (3) to determine whether the BOLD signals in the spinal cord are influenced by antinociceptive drugs.

\section{Materials and Methods}

Animal preparation and anesthesia. Functional MRI studies were conducted in 19 female Sprague Dawley rats weighing $260 \pm 10$ g. In 14 animals (Table 1, animals 1-14), fMRI was performed in the spinal cord, and in five animals, (Table 2, animals 15-19), fMRI was performed in medulla oblongata. Experiments were approved by the Stockholm Animal Ethics Committee.

The rats were first anesthetized with isoflurane $(2-2.5 \%)$ in a mixture of $40 \%$ oxygen and $60 \%$ nitrogen. Deeply anesthetized, rats were orally intubated and artificially ventilated using a rodent ventilator (7025; Ugo Basile, Comerio, Italy). An intravenous catheter (Neoflon 24 GA; Becton Dickinson, Stockholm, Sweden), flushed previously with heparinized saline, was placed in the tail vein for continuous administration of anesthetics and/or drugs. This catheter was connected to a micro-injection pump (CMA/100; Carnegie Medicine AB, Stockholm, Sweden). On each hindlimb, a pair of 28 gauge needle electrodes (Grass Telefactor, Slough, UK) was implanted subcutaneously 3-4 mm apart near the achilles tendon. The paired electrodes were connected to an electric pulse generator (Medres Medical Research GmbH, Cologne, Germany) to deliver pulsed currents of $0.5,1.0,1.5$, or $2.0 \mathrm{~mA}$ with $1 \mathrm{~ms}$ duration and a constant frequency of $3 \mathrm{~Hz}$.
Table 2. BOLD signals in medulla oblongata in response to weak $(0.5 \mathrm{~mA})$ electrical stimulation

\begin{tabular}{cll}
\hline $0.5 \mathrm{~mA} \alpha$-chloralose & & \\
\hline Animal & $\beta$ estimate & $\begin{array}{l}\text { Cluster } \\
\text { volume }\end{array}$ \\
\hline 15 & 3.66 & 2.20 \\
16 & 1.18 & 3.08 \\
17 & 0.69 & 1.76 \\
18 & 0.89 & 1.76 \\
19 & 1.81 & 1.76 \\
Mean & $\mathbf{1 . 6 6}$ & $\mathbf{2 . 1 1}$ \\
SEM & $\mathbf{\pm 0 . 7 4}$ & $\mathbf{0 0 . 2 6}$
\end{tabular}

$\beta$ estimate and cluster volume of the fMRI signal measured in the medulla oblongata in the slice with maximal intensity for animals $15-19$. Electric stimulation of the hindlimb was performed using $0.5 \mathrm{~mA}$ pulsed currents. A BOLD signal was detected in all animals.

To assure correct placement of the electrodes, a short sequence of current pulses of $1 \mathrm{~mA}$ was applied to each limb outside the magnet evoking light muscle twitches in the gastrocnemius muscle. Next, a bolus of $1.5 \mathrm{ml}$ of $\alpha$-chloralose $(40 \mathrm{mg} / \mathrm{ml}$ ) was applied through the tail vein catheter and $10 \mathrm{~min}$ later, the isoflurane was discontinued. Anesthesia was continued with $\alpha$-chloralose infusion at a rate of $20 \mathrm{mg} / \mathrm{kg} / \mathrm{h}$. Pancuronium bromide infusion was supplemented at a rate of $4 \mathrm{mg} / \mathrm{kg} / \mathrm{h}$ to achieve good muscle relaxation. The rat was placed in supine position on a custom-built rig in the spectrometer. The rectal temperature was monitored and body temperature maintained at $37 \pm 0.5^{\circ} \mathrm{C}$ by a warm-air system during the experiment.

MRI equipment and sequence. A $4.7 \mathrm{~T}$ spectrometer with a horizontal bore (Biospec Avance 47/40; Bruker, Karlsruhe, Germany) and a surface coil (Bruker BioSpin; T9510) for MR signal transmission and detection were used to scan the spinal cord. The surface coil had an inner diameter of $20 \mathrm{~mm}$, which is sufficient to see three complete vertebrae. The scanner was interfaced by a Linux workstation and appropriate software (Paravision version 3.0.2; Bruker). Before fMRI, high-resolution spin-echo rapid acquisition with relaxation enhancement (RARE) images in sagittal and horizontal planes were obtained to localize the lumbar enlargement of the spinal cord. The 13th rib was used as a landmark to locate the appropriate level (Fraidakis et al., 1998). Axial slices were also useful to confirm correct position because the width of the spinal cord reaches a maximum at the level of the lumbar enlargement. The lumbar spinal cord could also be distinguished from the thoracic and the sacral spinal 
cord by the characteristic shape of the gray matter, as described previously (Williams and Warwick, 1980).

Local shimming was performed at the region of interest using a pointresolved spectroscopy protocol. A slice package consisted of seven adjacent axial slices with $2.1 \mathrm{~mm}$ thickness and a field of view of $20 \mathrm{~mm}$. This was centered at vertebra L1 (Malisza et al., 2003). The same geometry was used both for the collection of fMRI data and anatomical high-resolution images. For fMRI, a single-shot EPI sequence was used. The parameters were selected to achieve the highest image quality with the shortest possible effective echo time (TE) [TE, $20 \mathrm{~ms}$; repetition time (TR), $1500 \mathrm{~ms}$; matrix size, $64 \times 64$, yielding an in-plane resolution of [0.31, 0.31$] \mathrm{mm}]$. For high-resolution anatomical MRI, a T2-weighted spin-echo RARE sequence was chosen (TE, $25 \mathrm{~ms}$; TR, $3000 \mathrm{~ms}$; RARE factor, 4; $256 \times 256$ matrix; 16 averages).

When fMRI was recorded from medulla oblongata, a $35 \mathrm{~mm}$ volume coil (Bruker) was used. Seven adjacent transverse slices with the slice thickness $2 \mathrm{~mm}$ and a field of view $30 \mathrm{~mm}$ were imaged using an EPI sequence (TR/TE, 1500/20 ms; matrix size, $64 \times 64)$. The image packages were centered through cerebellum and medulla oblongata.

Electrical stimulation and block design. For fMRI, block design with two or three conditions was used. The block design with three conditions, which was applied in 10 rats (Table 1), was asymmetric and consisted of rest $(\mathrm{R})$ periods and periods in which electric stimulation applied to the hindlimb using alternating high and low current stimulation. Briefly, 27 image packages obtained during a rest period were followed by 13 image packages obtained during high-level stimulation (HS) followed by 27 image packages during $\mathrm{R}$ and 13 image packages during low-level stimulation (LS). High-level stimulation was defined as current pulses of 2 $\mathrm{mA}$; low-level stimulation was defined as current pulses of $0.5,1.0$, or 1.5 $\mathrm{mA}$. The block interval was repeated 10 times. At the end of this session additional 27 image packages during R, 13 image packages during HS, and 13 image packages during $\mathrm{R}$ were added. This was done because the first 40 image packages were removed for the analysis to assure equilibrium of spin relaxation and steady-state conditions and the paradigm was aimed to end with a rest period. Hence, 867 slice packages were collected totally per session and 827 were used for the analysis. The paradigm can be described as follows: $10 \times(27 \mathrm{R}-13 \mathrm{HS}-27 \mathrm{R}-$ 13LS) + (27R-13HS-27R).

The block design with two conditions, which was used in four rats (Table 1, rat 11-14), was symmetric and consisted of 27 image packages acquired during $\mathrm{R}$ and 13 image packages acquired during HS. This was repeated 11 times and ended by an additional 27 image packages obtained during $\mathrm{R}$. The paradigm can be described as follows: $11 \times(27 \mathrm{R}-$ $13 \mathrm{HS})+27 \mathrm{R}$. For data analysis, the first block interval (40 image packages) was also ignored.

Each rat was exposed to three to five fMRI sessions, as indicated in Table 1. For each rat, this included one session in which $2 \mathrm{~mA}$ current pulses were applied using either the block design with two or the block design with three conditions. This session was performed under $\alpha$-chloralose anesthesia only. Thereafter, one session followed in which intravenous morphine (10 mg/kg; AstraZeneca, Sodertalje, Sweden) was applied $10 \mathrm{~min}$ before the fMRI session and using the identical block design as for the previous session. To evaluate a morphine effect, care was taken to use the identical block design for the sessions before morphine injection and under the influence of morphine. In nine animals, this was followed by an fMRI session in which naloxone ( $4 \mathrm{mg} / \mathrm{kg}$; Bristol-Myers Squibb, Wallingford, CT) was applied 5 min before the fMRI session. Again, an identical block design as in the previous session was used.

To study BOLD responses in medulla oblongata, currents of $0.5 \mathrm{~mA}$ were applied to either the right or the left hindlimb using a block design with two conditions consisting of 27 EPI image packages (seven slices each) acquired during rest and 13 EPI image packages (seven slices each) during stimulation that was repeated 10 times and ended with an additional 27 EPI image packages (seven slices each) at rest [27R-13S $+10 \times$ $(27 \mathrm{R}-13 \mathrm{~S})+27 \mathrm{R}]$, providing a total of 467 image packages consisting of totally 3269 images acquired in $11.7 \mathrm{~min}$.

After the fMRI experiments, animals were killed using an overdose of pentobarbital (120 mg/kg, i.v.).

Data processing. To evaluate the fMRI data, the software toolkit SPM2
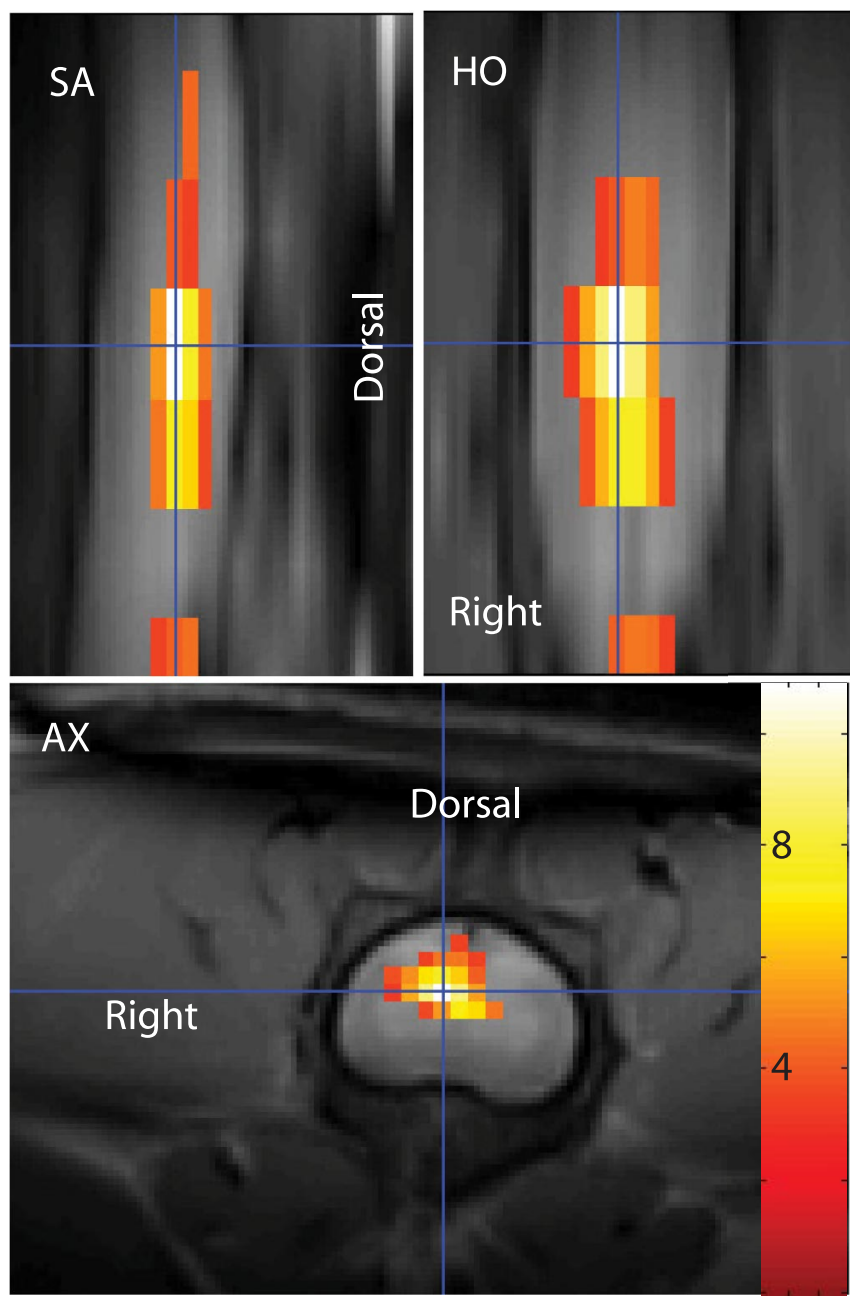

Figure 1. Three-dimensional reconstruction of a BOLD fMRI signal in the spinal cord during right hindlimb stimulation at $2 \mathrm{~mA}$. The parametric map is projected onto sagittal (SA), horizontal (HO), and axial ( $\mathrm{AX}$ ) views through the spinal cord. Right and dorsal sides are indicated. This displays size, shape, and location of the activated functional unit. The axial panel clearly shows that the fMRI signal is located dorsally and ipsilateral to the stimulation. The color scale indicates the $t$ statistics calculated by SPM software. Brighter and darker colors indicate higher and lower $t$ values, respectively.

(the Wellcome Department of Cognitive Neurology, London, UK) was used. All fMRI data were smoothed using a Gaussian kernel with a full width at half maximum that corresponds to approximately three times the in-plane voxel size. Smoothing has, according to the central limit theorem, the effect of rendering data more normally distributed, thereby increasing the validity of parametric statistical tests. A design matrix that described the stimulation paradigm was then created with SPM2. The square stimulation pattern (R-LS-R-LS or R-HS-R-HS) was convolved with the built-in hemodynamic response function in SPM2. A critical $t$ value for each voxel was calculated for the significance level of $p<0.001$, resulting in a statistical parametric map that was then mapped on to the high-resolution anatomical slice package.

To evaluate the effect of stimulation with different levels of current and the influence of drugs, the voxel with the highest significance (i.e., the voxel with the highest $t$ value) was located. The $\beta$ estimation that corresponded to the estimated contrast difference for this voxel was then calculated for all conditions in each animal. The $\beta$ estimate could be considered as a measure of the BOLD response amplitude. Also, activated voxels in the same slice were integrated to estimate the size of the activated area. The alteration of fMRI signal induced by morphine $(2 \mathrm{~mA}$ $\alpha$-chloralose $/ 2 \mathrm{~mA} \alpha$-chloralose plus morphine) and naloxone ( $2 \mathrm{~mA}$ $\alpha$-chloralose plus morphine $/ 2 \mathrm{~mA} \alpha$-chloralose plus morphine plus nal- 
A

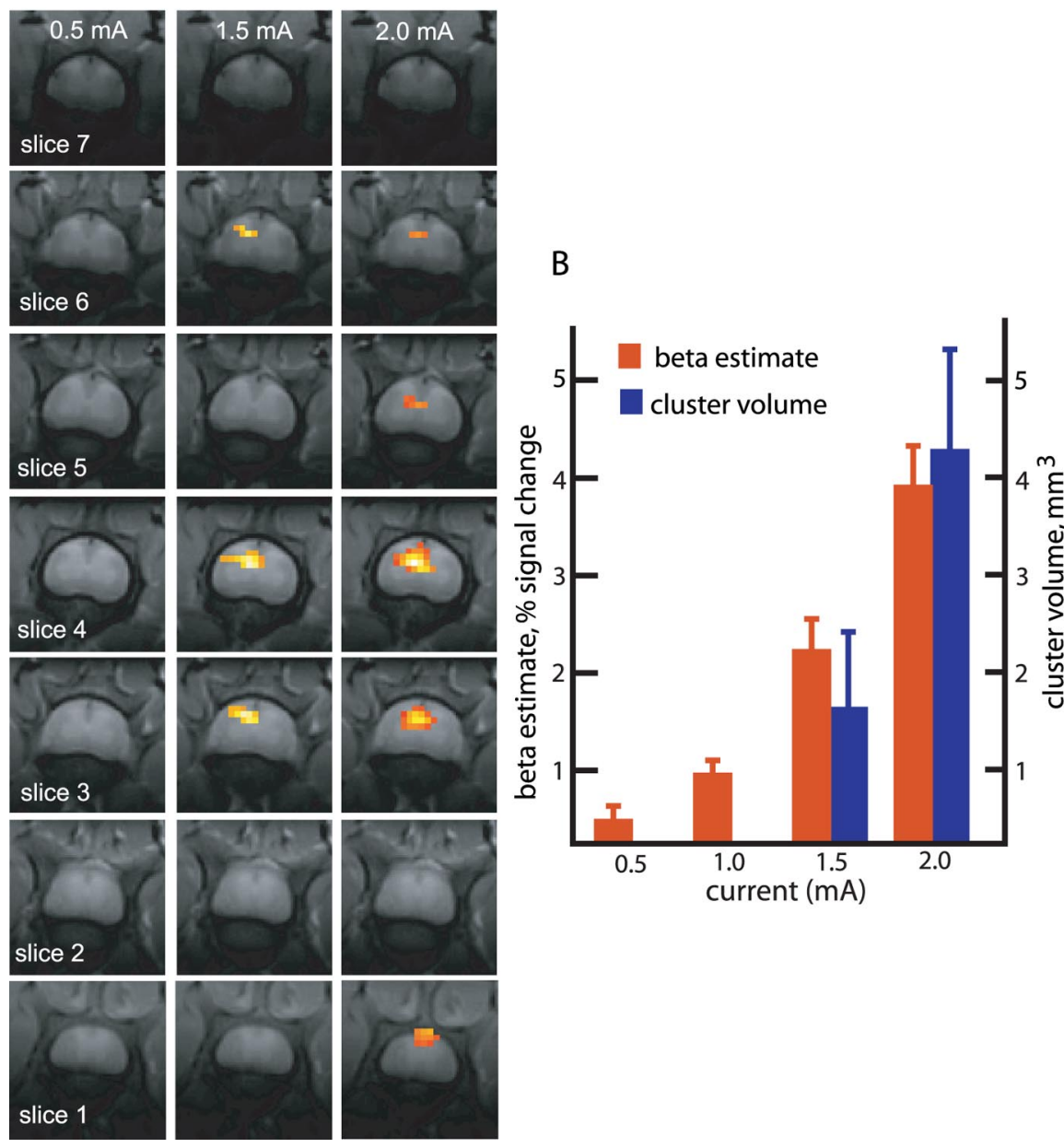

Figure 2. BOLD responses in the lumbar spinal cord in response to graded stimulation of the right hindlimb. $\boldsymbol{A}$, Individual axial slices are shown in vertical columns. Current pulses of $0.5,1.5$, or $2 \mathrm{~mA}$ were applied to the right hindlimb, and the parametric map of the BOLD signals is superimposed on the respective anatomic axial images. At $0.5 \mathrm{~mA}$, no significant activation was seen. BOLD signals are detected in three and five slices at 1.5 and $2 \mathrm{~mA}$, respectively. Orientation of the axial images is the same as in Figure 1. $\boldsymbol{B}$, Graph showing quantitative evaluation of BOLD signals by $\beta$ estimates and cluster volumes in response to current pulses of $0.5,1.0,1.5$, and $2.0 \mathrm{~mA}$. Pixels did not reach the selected statistical threshold of $p<0.001$ at 0.5 or $1.0 \mathrm{~mA}$. Both $\beta$ estimates $(p<0.001)$ and cluster volumes $(p<0.001)$ were significantly correlated to stimulus strength. Error bars represent SEM.

The strongest BOLD signal was seen at the level of vertebra L1, but some signal could also be seen in the immediately adjacent rostral and caudal levels. Using SPM, the spatial features of the BOLD signal in three projection planes (Fig. 1) were obtained, and this volume presumably outlines the synaptic field of the activated incoming sensory axons. Interestingly, 2 $\mathrm{mA}$ evoked strong and robust BOLD signals in the dorsal horn of the spinal cord, whereas 1.0 or $0.5 \mathrm{~mA}$ applied to the hindlimb did not evoke any BOLD response in the spinal cord. The statistical threshold for detection of BOLD signals was set to $p<0.001$. Figure 2 shows the fMRI result of an animal that was stimulated with 0.5 , 1.5 , or $2 \mathrm{~mA}$ current pulses. The area of the BOLD signal in corresponding slices was appreciably larger when $2 \mathrm{~mA}$ was applied than when $1.5 \mathrm{~mA}$ was applied. No BOLD signal was seen when $0.5 \mathrm{~mA}$ was applied.

The $\beta$ estimate was measured in corresponding slices, picking the pixel with the highest/most significant BOLD signal. The $\beta$ estimate describes the percentage increase of the image intensity during a test situation compared with the intensity at rest. As shown quantitatively in the plot in Figure 2, the $\beta$ estimate was clearly correlated to the intensity of the applied stimulus [Pearson's correlation coefficient of $\ln (\beta$ estimate $)$ vs $\mathrm{mA}$ stimulus $=0.905$; $p<0.001]$ and reached an average of $2.18 \%$ at $1.5 \mathrm{~mA}$ and $3.91 \%$ at $2.0 \mathrm{~mA}$ current pulses. At 0.5 and $1.0 \mathrm{~mA}$, the $\beta$ estimate was below 1\%. Quantification of the cluster volume in each animal, which was calculated from the number of activated pixels in the slice with the strongest BOLD signal, showed that there was a clear signal in response to $1.5 \mathrm{~mA}$ and a stronger re-

oxone) were calculated and the statistical significance was tested using the nonparametric Wilcoxon analysis. Pearson's correlation coefficient was calculated to establish an association between (1) cluster volume and $\beta$ estimate and (2) the logarithmic transformation of $\beta$ estimate or cluster volume and stimulus strength. A logarithmic transformation of the values was performed because the data points were better fitted with exponential function than with linear function. All values are given as mean \pm SEM.

\section{Results \\ BOLD signals in the spinal cord in response to graded hindlimb stimulation}

The results of the 14 individual rats used to study BOLD responses in the spinal cord and subjected to $0.5,1.0,1.5$, or $2 \mathrm{~mA}$ of electric current pulses to the hindlimb are summarized in $\mathrm{Ta}$ ble 1 . All animals showed a strong and robust fMRI signal at 2 $\mathrm{mA}$. The fMRI signal was predominantly situated in the dorsal and intermediate gray matter in lamina II-VI (Fig. 1). An fMRI signal was always seen in the ipsilateral gray matter but could also involve the contralateral side. In four animals, it was also tested to switch the stimulus from one hindpaw to the other, which flipped the location of the fMRI signal. sponse elicited by $2 \mathrm{~mA}$ [Pearson's correlation coefficient of ln(cluster volume) vs mA stimulus $=0.928 ; p<0.001]$ (Fig. 2). Validity of $\beta$ estimate and cluster volume measures were confirmed by the observation that cluster size and $\beta$ estimate were positively correlated [Pearson correlation of coefficient of $\ln (\beta$ estimate) vs $\ln ($ cluster volume $)=0.860 ; p<0.001]$.

\section{BOLD signals in medulla oblongata depict dorsal column pathway}

We observed robust fMRI signals in ipsilateral medulla oblongata and cerebellum in rats when $0.5 \mathrm{~mA}$ current pulses were applied to the hindlimbs (Fig. $3 B$ ). The location of the signal in dorsal medulla is compatible with the location of the cuneate and gracile nuclei (Paxinos and Watson, 2005). The $\beta$ estimate of the BOLD signal assessed in medulla oblongata during $0.5 \mathrm{~mA}$ current pulses was $1.66 \%$ and the mean cluster volume was $2.11 \mathrm{~mm}^{3}$ (Table 2).

\section{Morphine decreases the BOLD response in the spinal cord}

We next compared the BOLD response during $2 \mathrm{~mA}$ stimulation in animals before and after morphine injections. As shown in 
Figure 4, the fMRI signal was reduced under the influence of morphine. Three general features were observed. First, the size of the fMRI signal became smaller under the influence of morphine. Second, the maximal $t$ value of the BOLD signal was smaller. Third, quantitative analysis revealed that the mean $\beta$ estimate and the mean cluster volume of the BOLD responses were both significantly smaller after morphine than in control situations $(p<0.01)$. The specificity of the morphine effect was tested by naloxone. Treatment with naloxone led to a significant recovery of the BOLD signal from the morphine-induced diminution $(p<0.05)$.

\section{Discussion}

In this study, we used an EPI sequence to detect BOLD contrast changes in the rat spinal cord and brain in response to sensory stimulation of a hindlimb. We demonstrate quantitatively, measuring both $\beta$ estimates and cluster volume, that it is possible to detect and differentiate between the major synaptic relay stations of the spinothalamic and the dorsal column somatosensory pathways. BOLD responses in thalamus and the cerebral cortex were documented previously (Spenger et al., 2000; Hofstetter et al., 2003). Functional MRI can thus be used to monitor all major synaptic relay stations along the neuroaxis as sensory input from the periphery enters the spinal cord and ascends through the CNS to reach cortex cerebri.

Gradient echo has most often been used in fMRI because of its high sensitivity in detecting BOLD effects (Bandettini et al., 1994; Stroman et al., 2001). However, application of this fMRI technique to the rodent spinal cord has not been reported before. This is probably because the technique is highly sensitive to local field inhomogeneity, which is prominent in the spinal cord as a result of flow of CSF, magnetic property differences between the spinal cord and the surrounding vertebral column, and the small crosssectional dimensions of the spinal cord itself. More recently, another fMRI contrast mechanism, signal enhancement by extravascular water protons, was introduced and used in spinal fMRI of humans and rats with spin echo-based pulses (Stroman et al., 2002, 2003a,b, 2005b; Majcher et al., 2006). Another early study of spinal fMRI in rats also used a spin echo based technique (Porszasz et al., 1997).

Here, we demonstrate that use of a gradient echo sequence in spinal cord fMRI is a useful option that should allow wider application of spinal cord fMRI in rodents. EPI sequence protocols are also common in fMRI because they reduce image acquisition time and thereby improve temporal resolution and statistical power. Our current protocol resulted in robust fMRI signals and was highly sensitive. We could generate 3269 images in $\sim 11 \mathrm{~min}$, or close to five images per second. This high number of images contributed to solid statistic correlations. Hence, each session of each individual could be evaluated individually. This is not always the case when sequences with lower temporal resolution are used. The possibility for analysis of individual sessions is also important, because a group analysis with SPM is only possible for the rat brain (Schweinhardt et al., 2003) but not for the rat spinal cord because of the present lack of an appropriate spinal cord template. SPM nevertheless allows for the analysis of the fMRI signal similar to a recently improved method for spinal functional MRI with large volume coverage of the spinal cord (Stroman et al., 2005a) in three dimensions. This allows focusing through the spinal cord and virtually reslicing the image package to view results in three separate dimensions simultaneously. We demonstrate that the spinal cord fMRI signal is spindle-shaped along the longitudinal axis of the spinal cord and extends over several segments. We also found that neuronal activity, as reflected by BOLD signals, which was localized to the expected ipsilateral dorsal horn of the spinal cord, and that the BOLD signal strength ( $\beta$ estimate as well as cluster volume) was closely correlated to the strength of the applied electrical hindlimb stimulation.

Our study provides evidence that the applied fMRI method is valid and sensitive. This is supported by (1) the robustness of the BOLD signals, (2) the significant correlation between stimulus intensities and responses, (3) the match of BOLD signals with known neuroanatomy, and (4) expected effects of morphine and naloxone. However, it should be noted that gradient echo EPI might account for some spatial distortion depending on magnetic field strength and inhomogeneity as mentioned above. This could result in some displacement of statistic parametric maps when projected onto spin echo images. Nevertheless, spin echo images were preferred for projection in Figures 1-3 because of superior contrast and resolution to describe anatomical location of the signals.

The high sensitivity allowed us also to distinguish fMRI signals 


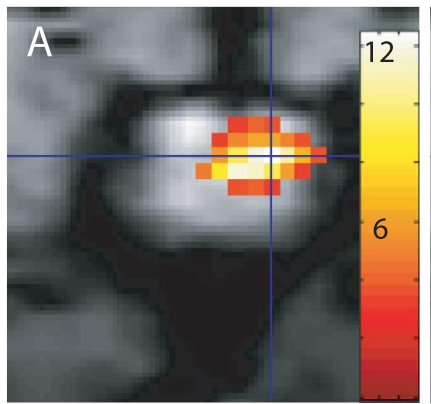

C

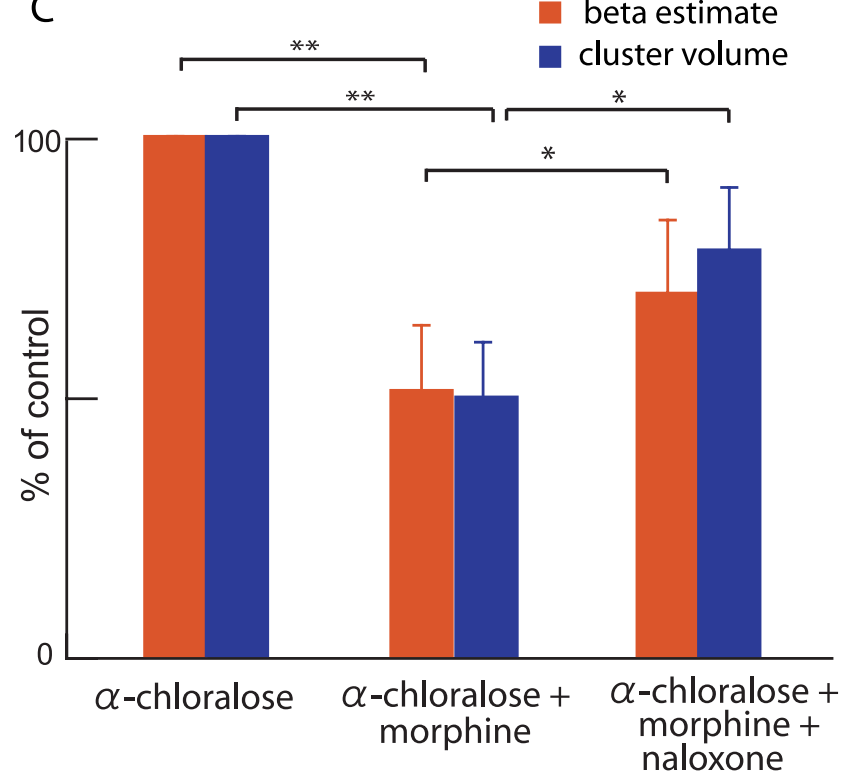

Figure 4. Effects of morphine and naloxone on BOLD fMRI signals in the lumbar spinal cord. $A$, Representative fMRI showing BOLD signals in response to left hindlimb stimulation at $2 \mathrm{~mA}$ before morphine injection. $B, B O L D$ response is decreased after morphine injection. The parametric statistical maps of BOLD responses were superimposed on axial EPI images. Orientation of the images is the same as in the axial slice in Figure 1 . The color scale in the figures indicates the $t$ statistics as explained in Figure 1. C, There is significant decrease of both $\beta$ estimate and cluster volume after morphine injection. These effects were counteracted by naloxone, causing the BOLD response as reflected by both $\beta$ estimates and cluster volumes to recover significantly. ${ }^{*} p<0.05 ;{ }^{* *} p<0.01$; significances calculated from original values given in Table 1. Error bars represent SEM.

that were evoked by different sensory pathways in individual animals. Stronger stimulation of the hindlimb produced BOLD signals in the dorsal horn, whereas weaker stimulation did not cause activation in the dorsal horn, but activated nuclei in medulla oblongata. This difference faithfully reflects the established neuroanatomy of painful versus nonpainful pathways (i.e., the difference between the spinothalamic pain pathway and the dorsal column somatosensory pathway) (Williams and Warwick, 1980). Fibers of the spinothalamic tract propagate information from receptors signaling pain. Cutaneous and other nociceptors are supplied by finely myelinated $(\mathrm{A} \delta$ ) and unmyelinated (C) fibers. These axons pass through the dorsal roots into the dorsolateral fasciculus (Lissauer's tract) and end mainly in the superficial layers of the dorsal horn. In line with this, we found fMRI activity responding to the higher current and probably related to noxious stimulation in these areas. The large, myelinated $(\mathrm{A} \beta)$ axons of the lumbar primary afferents mediating touch do not predominantly synapse in the dorsal horn but ascend in the dorsal columns to medulla oblongata, where they synapse with secondary sensory neurons in the gracile (hindlimb afferents) and cuneate (forepaw afferents) nuclei. These pathways relay non-noxious stimulation, well in line with the fact that lower current stimuli produced robust fMRI signals in medulla oblongata.

We also demonstrate that the prominent BOLD signals in the dorsal horn of the spinal cord can be effectively attenuated by morphine. This effect was partially reversed by the morphine antagonist naloxone. Thus, although BOLD fMRI has been shown previously to detect effects of morphine in the rodent brain (Chang and Shyu, 2001), we now show by fMRI that the morphine effect is prominent already at the spinal cord level. A robust morphine effect can be located to the level of the first synaptic relay station for noxious stimuli in the dorsal horn of the lumbar spinal cord. This is relevant to the interpretation of drug effects and the technique should become valuable in the development and in vivo testing of novel drug candidates for the treatment of pain. Effects of drugs such as morphine and naloxone on fMRI signals should, however, be interpreted with caution. One parameter that may affect the magnitude of BOLD responses is abrupt changes of blood pressure (Kalisch et al., 2001; Tuor et al., 2002). Gradual changes that remain within the range of effective cerebral autoregulation are less likely to be associated with BOLD signal alterations (Zaharchuk et al., 1999; Shah et al., 2005).

We conclude that fMRI, using the current protocol and the BOLD response, is well suited for studies of how various sensory stimuli evoke responses in the spinal cord and medulla oblongata. We find that BOLD responses to graded stimulations of a hindlimb are faithfully elicited at the synaptic relay stations of the spinothalamic and dorsal column somatosensory pathways. Therefore, increased activity of all major synaptic relay stations conveying sensory information from the periphery to the cerebellar and cerebral cortices can be detected in rats using fMRI. Rat spinal cord fMRI should become useful in assessing novel experimental protective and repair strategies for spinal cord injury as well as in studies of novel drugs for the treatment of pain.

\section{References}

Austin VC, Blamire AM, Allers KA, Sharp T, Styles P, Matthews PM, Sibson NR (2005) Confounding effects of anesthesia on functional activation in rodent brain: a study of halothane and alpha-chloralose anesthesia. NeuroImage 24:92-100.

Bandettini PA, Wong EC, Jesmanowicz A, Hinks RS, Hyde JS (1994) Spinecho and gradient-echo EPI of human brain activation using BOLD contrast: a comparative study at $1.5 \mathrm{~T}$. NMR Biomed 7:12-20.

Bock C, Krep H, Brinker G, Hoehn-Berlage M (1998) Brainmapping of alpha-chloralose anesthetized rats with $\mathrm{T} 2^{\star}$-weighted imaging: distinction between the representation of the forepaw and hindpaw in the somatosensory cortex. NMR Biomed 11:115-119.

Chang C, Shyu BC (2001) A fMRI study of brain activations during nonnoxious and noxious electrical stimulation of the sciatic nerve of rats. Brain Res 897:71-81.

Dijkhuizen RM, Singhal AB, Mandeville JB, Wu O, Halpern EF, Finklestein SP, Rosen BR, Lo EH (2003) Correlation between brain reorganization, ischemic damage, and neurologic status after transient focal cerebral ischemia in rats: a functional magnetic resonance imaging study. J Neurosci 23:510-517.

Elfgren C, van Westen D, Passant U, Larsson EM, Mannfolk P, Fransson P (2006) fMRI activity in the medial temporal lobe during famous face processing. NeuroImage 30:609-616.

Fraidakis M, Klason T, Cheng H, Olson L, Spenger C (1998) Highresolution MRI of intact and transected rat spinal cord. Exp Neurol 153:299-312.

Fransson P, Kruger G, Merboldt KD, Frahm J (1998) Temporal characteristics of oxygenation-sensitive MRI responses to visual activation in humans. Magn Reson Med 39:912-919.

Fransson P, Kruger G, Merboldt KD, Frahm J (1999) Temporal and spatial MRI responses to subsecond visual activation. Magn Reson Imaging $17: 1-7$. 
Heeger DJ, Ress D (2002) What does fMRI tell us about neuronal activity? Nat Rev Neurosci 3:142-151.

Hofbauer RK, Fiset P, Plourde G, Backman SB, Bushnell MC (2004) Dosedependent effects of propofol on the central processing of thermal pain. Anesthesiology 100:386-394.

Hofbauer RK, Olausson HW, Bushnell MC (2006) Thermal and tactile sensory deficits and allodynia in a nerve-injured patient: a multimodal psychophysical and functional magnetic resonance imaging study. Clin J Pain 22:104-108.

Hofstetter CP, Schweinhardt P, Klason T, Olson L, Spenger C (2003) Numb rats walk-a behavioural and fMRI comparison of mild and moderate spinal cord injury. Eur J Neurosci 18:3061-3068.

Hofstetter CP, Holmstrom NA, Lilja JA, Schweinhardt P, Hao J, Spenger C, Wiesenfeld-Hallin Z, Kurpad SN, Frisen J, Olson L (2005) Allodynia limits the usefulness of intraspinal neural stem cell grafts; directed differentiation improves outcome. Nat Neurosci 8:346-353.

Hwang JH, Wu CW, Chou PH, Liu TC, Chen JH (2005) Hemispheric difference in activation patterns of human auditory-associated cortex: an FMRI study. ORL J Otorhinolaryngol Relat Spec 67:242-246.

Kalisch R, Elbel GK, Gossl C, Czisch M, Auer DP (2001) Blood pressure changes induced by arterial blood withdrawal influence bold signal in anesthesized rats at 7 Tesla: implications for pharmacologic MRI. NeuroImage 14:891-898.

Keilholz SD, Silva AC, Raman M, Merkle H, Koretsky AP (2004) Functional MRI of the rodent somatosensory pathway using multislice echo planar imaging. Magn Reson Med 52:89-99.

Majcher K, Tomanek B, Jasinski A, Foniok T, Stroman PW, Tuor UI, Kirk D, Hess G (2006) Simultaneous functional magnetic resonance imaging in the rat spinal cord and brain. Exp Neurol 197:458-464.

Malisza KL, Stroman PW, Turner A, Gregorash L, Foniok T, Wright (2003) A functional MRI of the rat lumbar spinal cord involving painful stimulation and the effect of peripheral joint mobilization. J Magn Reson Imaging 18:152-159.

Mukamel R, Gelbard H, Arieli A, Hasson U, Fried I, Malach R (2005) Coupling between neuronal firing, field potentials, and FMRI in human auditory cortex. Science 309:951-954.

Niessing J, Ebisch B, Schmidt KE, Niessing M, Singer W, Galuske RA (2005) Hemodynamic signals correlate tightly with synchronized gamma oscillations. Science 309:948-951.

Ogawa S, Menon RS, Tank DW, Kim SG, Merkle H, Ellermann JM, Ugurbil K (1993) Functional brain mapping by blood oxygenation level-dependent contrast magnetic resonance imaging. A comparison of signal characteristics with a biophysical model. Biophys J 64:803-812.

Olausson H, Charron J, Marchand S, Villemure C, Strigo IA, Bushnell MC (2005) Feelings of warmth correlate with neural activity in right anterior insular cortex. Neurosci Lett 389:1-5.

Pastor MA, Macaluso E, Day BL, Frackowiak RS (2006) The neural basis of temporal auditory discrimination. NeuroImage 30:512-520.

Paxinos G, Watson C (2005) The rat brain in stereotaxic coordinates-the new coronal set, Ed 5. London: Academic.

Porszasz R, Beckmann N, Bruttel K, Urban L, Rudin M (1997) Signal changes in the spinal cord of the rat after injection of formalin into the hindpaw: characterization using functional magnetic resonance imaging. Proc Natl Acad Sci USA 94:5034-5039.

Schweinhardt P, Fransson P, Olson L, Spenger C, Andersson JL (2003) A template for spatial normalisation of MR images of the rat brain. J Neurosci Methods 129:105-113.

Seung Y, Kyong JS, Woo SH, Lee BT, Lee KM (2005) Brain activation during music listening in individuals with or without prior music training. Neurosci Res 52:323-329.

Shah YB, Haynes L, Prior MJ, Marsden CA, Morris PG, Chapman V (2005) Functional magnetic resonance imaging studies of opioid receptormediated modulation of noxious-evoked BOLD contrast in rats. Psychopharmacology 180:761-773.

Silva AC, Lee SP, Yang G, Iadecola C, Kim SG (1999) Simultaneous blood oxygenation level-dependent and cerebral blood flow functional magnetic resonance imaging during forepaw stimulation in the rat. J Cereb Blood Flow Metab 19:871-879.

Spenger C, Josephson A, Klason T, Hoehn M, Schwindt W, Ingvar M, Olson L (2000) Functional MRI at 4.7 tesla of the rat brain during electric stimulation of forepaw, hindpaw, or tail in single- and multislice experiments. Exp Neurol 166:246-253.

Stroman PW, Krause V, Frankenstein UN, Malisza KL, Tomanek B (2001) Spin-echo versus gradient-echo fMRI with short echo times. Magn Reson Imaging 19:827-831.

Stroman PW, Krause V, Malisza KL, Frankenstein UN, Tomanek B (2002) Extravascular proton-density changes as a non-BOLD component of contrast in fMRI of the human spinal cord. Magn Reson Med 48:122-127.

Stroman PW, Tomanek B, Krause V, Frankenstein UN, Malisza KL (2003a) Functional magnetic resonance imaging of the human brain based on signal enhancement by extravascular protons (SEEP fMRI). Magn Reson Med 49:433-439.

Stroman PW, Malisza KL, Onu M (2003b) Functional magnetic resonance imaging at 0.2 Tesla. NeuroImage 20:1210-1214.

Stroman PW, Kornelsen J, Bergman A, Krause V, Ethans K, Malisza KL, Tomanek B (2004) Noninvasive assessment of the injured human spinal cord by means of functional magnetic resonance imaging. Spinal Cord 42:59-66.

Stroman PW, Kornelsen J, Lawrence J (2005a) An improved method for spinal functional MRI with large volume coverage of the spinal cord. J Magn Reson Imaging 21:520-526.

Stroman PW, Kornelsen J, Lawrence J, Malisza KL (2005b) Functional magnetic resonance imaging based on SEEP contrast: response function and anatomical specificity. Magn Reson Imaging 23:843-850.

Thompson JK, Peterson MR, Freeman RD (2003) Single-neuron activity and tissue oxygenation in the cerebral cortex. Science 299:1070-1072.

Tuor UI, Malisza K, Foniok T, Papadimitropoulos R, Jarmasz M, Somorjai R, Kozlowski P (2000) Functional magnetic resonance imaging in rats subjected to intense electrical and noxious chemical stimulation of the forepaw. Pain 87:315-324.

Tuor UI, McKenzie E, Tomanek B (2002) Functional magnetic resonance imaging of tonic pain and vasopressor effects in rats. Magn Reson Imaging 20:707-712.

Williams PL, Warwick R (1980) Gray's anatomy, Ed 36. Edinburgh: Churchill Livingstone.

Zaharchuk G, Mandeville JB, Bogdanov Jr AA, Weissleder R, Rosen BR, Marota JJ (1999) Cerebrovascular dynamics of autoregulation and hypoperfusion. An MRI study of CBF and changes in total and microvascular cerebral blood volume during hemorrhagic hypotension. Stroke 30: 2197-2204. 